\title{
The Kinetics of Synaptic Vesicle Reacidification at Hippocampal Nerve Terminals
}

\author{
Pradeep P. Atluri and Timothy A. Ryan \\ Department of Biochemistry, Weill Medical College of Cornell University, New York, New York 10021
}

\begin{abstract}
After exocytosis, synaptic vesicles are recycled locally in the synaptic terminal and are refilled with neurotransmitter via vesicular transporters. The biophysical mechanisms of refilling are poorly understood, but it is clear that the generation of a proton gradient across the vesicle membrane is crucial. To better understand the determinants of vesicle refilling, we developed a novel method to measure unambiguously the kinetics of synaptic vesicle reacidification at individual synaptic terminals. Hippocampal neurons transfected with synapto-pHluorin $(\mathrm{SpH})$, a synaptic vesicle-targeted lumenal GFP (green fluorescent protein), whose fluorescence is quenched when protonated $\left(\mathrm{pK}_{\mathrm{a}} \sim 7.1\right)$, were rapidly surface-quenched immediately after trains of repetitive electrical stimulation. The recently endocytosed alkaline pool of $\mathrm{SpH}$ is protected from such surface quenching, and its fluorescence decay reflects reacidification kinetics. These measurements indicate that, after compensatory endocytosis, synaptic vesicles reacidify with first-order kinetics $(\tau \sim 4-5 \mathrm{~s})$ and that their rate of reacidification is subject to slowing by increased external buffer.
\end{abstract}

Key words: hippocampus; fluorescence microscopy; synaptic vesicle release; optical imaging; synaptic transmission; synaptic

\section{Introduction}

In newly endocytosed synaptic vesicles, a proton electrochemical gradient (Grabe and Oster, 2001) produced by the V-type $\mathrm{H}^{+}$ATPase2 (Nishi and Forgac, 2002) powers transport of neurotransmitter molecules into the vesicle lumen (Liu and Edwards, 1997; Fremeau et al., 2004). Previous studies indicate that quantal size and release probability may be related to steady-state vesicle pH (Wolosker et al., 1996; Pothos et al., 2002). In principle, postendocytic reacidification kinetics could influence short-term synaptic plasticity by modulation of the function of vesicular proteins involved in exocytosis or by limiting vesicle refilling speed.

Despite the potential importance of reacidification kinetics, technical limitations have hampered direct measurements in intact synapses. Experiments using synapto-pHluorin $(\mathrm{SpH})$ (Miesenbock et al., 1998) in our laboratory suggested that, after trains of stimulation, vesicles reacidify with a time constant at most on the order of seconds (Sankaranarayanan and Ryan, 2000). SpH was used recently with single action potential (AP) stimulation to measure rates of endocytosis/reacidification (Gandhi and Stevens, 2003). The authors found that SpH fluorescence transients, thought to represent individual vesicle fusion, uptake,

Received 0ct. 17, 2005; revised Jan. 11, 2006; accepted Jan. 12, 2006.

This work was supported by the National Institutes of Health (T.A.R.), the Hirschl Trust (T.A.R.), and the Charles H. Revson Foundation (P.P.A.). We thank James Rothman for kindly providing the superecliptic synapto-pHluorin construct, Tomás Fernández-Alfonso for careful reading of this manuscript, members of the Ryan laboratory for useful discussions, and Wayne Yan for technical assistance.

Correspondence should be addressed to Timothy A. Ryan, Department of Biochemistry, Weill Medical College of Cornell University, 1300 York Avenue, New York, NY 10021. E-mail: taryan@med.cornell.edu.

P. P. Atluri's present address: Department of Psychiatry, NewYork-Presbyterian Hospital, Weill Cornell Medical Center, 525 East 68th Street, NY, New York 10021.

DOI:10.1523/JNEUROSCI.4425-05.2006

Copyright $\odot 2006$ Society for Neuroscience $\quad$ 0270-6474/06/262313-08\$15.00/0 and acidification, can decay very rapidly $(<500 \mathrm{~ms})$. However, whether such rapid reacidification occurs after trains of AP remains unknown.

Here, we use repetitive electrical stimulation of $\mathrm{SpH}-$ transfected, cultured hippocampal neurons to measure precisely postendocytic reacidification kinetics. The poststimulus decay of $\mathrm{SpH}$ fluorescence ordinarily reflects a combination of the kinetics of both endocytosis and subsequent reacidification. To overcome this problem, we used pulse-pressure application of impermeant acid to isolate the kinetics of reacidification from that of endocytosis. The amplitudes of surface-quenched $\mathrm{SpH}$ fluorescence transients allowed us to estimate the dynamics of the quenchprotected, "alkaline pool" of vesicles. Deconvolution was then used to estimate the time course of endocytosis after brief stimulus trains such as those that lead to exocytosis of the readily releasable pool (RRP) of vesicles.

\section{Materials and Methods}

Preparation and solutions. On postnatal day 2, Sprague Dawley rat hippocampi (Ca3-Ca1 region) were dissected, dissociated, and plated on poly-ornithine-coated glass coverslips, as described previously (Ryan, 1999). On postnatal day 6 or 7 , neurons were transfected with superecliptic $\mathrm{SpH}$ as described previously (Sankaranarayanan and Ryan, 2000). Experiments were performed 5-20 d after transfection, and coverslips were mounted in a laminar-flow perfusion and stimulation chamber. Cells were perfused continuously at room temperature $\left(25^{\circ} \mathrm{C}\right)$. Control Tyrode's solution contained (in mM) $119 \mathrm{NaCl}, 2.5 \mathrm{KCl}, 2 \mathrm{CaCl}_{2}, 2$ $\mathrm{MgCl}_{2}, 25 \mathrm{HEPES}$, and 30 glucose; osmolarity was adjusted to $300 \mathrm{mOsm}$ with sucrose, $\mathrm{pH} 7.40$, with $\mathrm{NaOH}$. In addition, all solutions contained $10 \mu \mathrm{M}$ 6-cyano-7-nitroquinoxaline-2,3-dione (Research Biochemicals, Natick, MA), and $50 \mu \mathrm{M}$ D,L-2-amino-5-phosphonovaleric acid (Research Biochemicals). In bafilomycin A1 (Baf; Calbiochem, San Diego, CA) experiments, Baf (stored in frozen aliquots at $200 \mu \mathrm{M}$ in DMSO) was diluted to $1 \mu \mathrm{M}$ in $500 \mu \mathrm{l}$ of control Tyrode's and added dropwise to the preparation for $30 \mathrm{~s}$ before washout with Baf-free Tyrode's perfusion. 
Because Baf causes a slow alkalinization of internal vesicles (Sankaranarayanan and Ryan, 2001), it was important to start the trial soon $(<90 \mathrm{~s})$ after Baf application. In buffer experiments, glucose was lowered to 10 mM. HEPES was used at 5, 25, or $40 \mathrm{~mm}$. In Tris experiments, $\mathrm{NaCl}$ was reduced to $91 \mathrm{~mm}$; this led to a reduction in size of $\mathrm{SpH}$ responses; thus, longer trains $(300 \mathrm{AP}, 10 \mathrm{~Hz})$ were used to compensate. Tris base was used at 10, 20, or $50 \mathrm{~mm}, \mathrm{pH}$ 7.40, with $\mathrm{HCl}$. Attempts to use Tris-base concentration of $100 \mathrm{~mm}$ were unsuccessful, because they led to solutions with osmolarity of $>360$ mOsm after $\mathrm{pH}$ titration. $\mathrm{SpH}$ signals were diminished in this high-osmolarity solution, and cells did not survive for long.

Line-scan fluorescence measurements. $\mathrm{SpH}$ fluorescence from individual boutons was measured using a custom-built laser confocal-imaging system in line-scan mode, through a $40 \times 1.3$ numerical aperture Zeiss (Oberkochen, Germany) Fluar objective. An argon ion laser was used to illuminate the sample at $488 \mathrm{~nm}$; emitted light was passed through a $498-538 \mathrm{~nm}$ bandpass filter and detected with a photomultiplier tube. Custom software was used to acquire and store fluorescence measurements. Cells were stimulated through platinum-iridium field electrodes located at the inlet and outlet of the perfusion chamber, using $1 \mathrm{~ms}$ pulses and a field of $10 \mathrm{~V} / \mathrm{cm}$. Boutons from SpH-transfected cells that responded (>20\% increase in fluorescence) to stimuli of $60 \mathrm{AP}$ at $20 \mathrm{~Hz}$ were selected for study. In quenching experiments, only responding boutons that were readily (i.e., rapidly, deeply, and reliably) quenched by 300 $\mathrm{ms}$ test pulses of acidic solution were studied. Sampling intervals ranged from $21 \mathrm{~ms}$ (to optimize puffer position) to $346 \mathrm{~ms}$ (to minimize bleaching).

Measurements of reacidification kinetics. To isolate $\mathrm{SpH}$ fluorescence from recently endocytosed synaptic vesicles, surface $\mathrm{SpH}$ was rapidly quenched by pulse-pressure application (PicoSpritzer II; General Valve, Fairfield, NJ) of impermeant acid. The puffer pipette had a tip diameter of $5-10 \mu \mathrm{m}$ and was generally positioned $30-100 \mu \mathrm{m}$ (in the horizontal plane) from the target bouton and $30-50 \mu \mathrm{m}$ above. The puffer pipette was aimed directly at the target bouton, but high perfusion velocity (2-3 $\mathrm{ml} / \mathrm{min} ; 150-300 \mu \mathrm{l}$ chamber volume) limited exposure of the target cell to acidic solution leaking from the puffer pipette. In most experiments, the puffer pipette contained MES-buffered (pKa 6.1, 45 mM) Tyrode's solution, $\mathrm{pH} 5.25$. We found that neurons survived only a few repeated MES puffs after Baf application. HEPES-buffered ( $25 \mathrm{~mm}$ ) Tyrode's, $\mathrm{pH}$ 5.25 , was better tolerated and was used in the puffer pipette for Baf experiments. HEPES-buffered quenching gave reacidification kinetics identical to that obtained in MES. These buffers appear adequate for both quenching the surface and maintaining the low $\mathrm{pH}$ after endocytosis, because quenching in bafilomycin showed almost constant fluorescence in the poststimulus quench period (see Fig. $2 \mathrm{~A}$ ).

If the quench is adequate, the time course of fluorescence decay during the quench period should not be sensitive to vesicles endocytosed during the quench period, because these vesicles should be already acidic and should not contribute to the time-varying signal. However, turbulent mixing of the acidic quench solution with the alkaline bath perfusion might lead to contamination of the reacidification signal by fluorescence from ongoing endocytosis of partially quenched vesicles during the quench period. Thus, it was important to optimize the acid quench. Before most trials, the acid quench depth and rapidity of onset were probed with brief $(300 \mathrm{~ms})$ pulses and optimized by slightly repositioning the puffer pipette or adjusting the amplitude of the pressure pulse. Control experiments (data not shown) indicated that, after optimization, the depth of quench achieved during the pressure pulse was equivalent to that achieved by bath application of Tyrode's solution, pH 5.5. Some of the boutons examined could not be adequately quenched, perhaps because of glial barriers to diffusion and convection, which may interfere with rapid access of the acid solution to the bouton.

Alkaline pool dynamics. We define the "alkaline pool" as recently endocytosed vesicles alkaline with respect to the $\mathrm{pH}$ of resting vesicles (i.e., $\mathrm{pH} \sim 5.5)$. The "size" of the alkaline pool, as reported by SpH fluorescence during the quench period, is a sum over recently endocytosed vesicles weighted by the instantaneous fluorescence of each vesicle.

We studied alkaline pool dynamics by varying $\Delta t$, the time between onset of the $1 \mathrm{~s}, 40 \mathrm{~Hz}$ stimulus and the beginning of a $15 \mathrm{~s}$ acid quench.
Five to ten trials were performed on each bouton. To minimize acid exposure and prolong tissue viability, we omitted the $5 \mathrm{~s}$ prequench from these experiments. However, the quench was optimized before each trial (as explained above). For each bouton, the alkaline pool amplitude for a particular $\Delta t$ was calculated as the difference between the average fluorescence of five points (sampling interval, $346 \mathrm{~ms}$ ) at the beginning and end of the quench.

Because the alkaline pool plots from different boutons have different peak times, it was not clear how best to summarize their time courses. Figure $3 B$ (bottom) shows a simple average of the alkaline pool amplitudes (see above) without normalization. We elected to fit the plots of alkaline pool dynamics for each bouton to an $\alpha$ function with a delay term (see Fig. $3 B$, top). The fit parameters for each bouton studied were averaged and used to arrive at a "mean" $\alpha$ function (i.e., one with mean parameters). This method of averaging the alkaline pool time courses gave a mean $\alpha$ function (see Figs. 4,5 ) with parameters similar to those from a fit to the simple average of the data (see Fig. $3 B$, bottom). The choice of the $\alpha$ function for curve fitting was somewhat arbitrary, but convenient. We do not imply that there are not many other ways to parameterize these curves.

Deconvolution. If endocytosis and reacidification are the only contributants to alkaline pool amplitude, and if they are linear timeinvariant processes, we can recover the endocytosis time course by deconvolution of reacidification from the alkaline pool time course. A linear time-invariant process is one in which (1) the amplitude of the response (intraquench fluorescence transient) varies linearly with the amplitude of the input (endocytosis); and (2) the response to a particular input does not depend on the time of that input. Deconvolution was calculated by dividing the fast Fourier transform (FFT) of the alkaline pool time course by the FFT of the reacidification time course and then performing an inverse FFT on the result.

After a $1 \mathrm{~s}, 40 \mathrm{~Hz}$ stimulus, there was a slight increase in the time constant of reacidification with increasing stimulus to quench intervals (data not shown). One interpretation is that the impulse response is not strictly time invariant; instead, it slightly slows with longer poststimulus times. Another possibility is that there is a small, slower second component to reacidification, which we do not resolve because of our limited (15s) quench window. Because endocytosis rates decline with poststimulus time, the mean delay between vesicle entry and the quench will increase for larger stimulus-quench intervals. The greater mean delay would be expected to accentuate the contribution of a second, slower component of reacidification.

Simulation. We numerically simulated alkaline pool dynamics using a fourth-order Runge-Kutta algorithm implemented in IGOR Pro 4 (WaveMetrics, Lake Oswego, OR) and assumed that exocytosis proceeded at a constant velocity during the stimulus and was zero otherwise. Similarly, we assumed that, before exocytosis, endocytosis was zero. We also assumed that endocytosis and reacidification during the poststimulus period were first-order processes whose velocities were proportional to the amount of $\mathrm{SpH}$ in the surface and alkaline pools, respectively.

\section{Results}

We used laser-confocal microscopy of cultured hippocampal neurons transfected with $\mathrm{SpH}$ to monitor the lumenal $\mathrm{pH}$ of synaptic vesicles in individual presynaptic terminals during and after brief trains of electrical stimuli. $\mathrm{SpH}$ is a $\mathrm{pH}$-sensitive $\left(\mathrm{pK}_{\mathrm{a}}\right.$ $\sim 7.1$ ) green fluorescent protein (GFP) conjugated to the lumenal domain of VAMP2 (vesicle-associated membrane protein)/ synaptobrevin, a v-SNARE (soluble $N$-ethylmaleimide-sensitive factor attached protein receptor) located in synaptic vesicle membranes (Miesenbock et al., 1998). In fully acidified ( $\mathrm{pH} \sim$ 5.5) synaptic vesicles of a resting bouton, $\mathrm{SpH}$ molecules spend $\sim 97 \%$ of the time in the quenched, proton-bound state (Sankaranarayanan and Ryan, 2000). Electrical stimulation triggers synaptic vesicles to fuse with the plasma membrane, in which $\mathrm{SpH}$ accumulates (Fernandez-Alfonso and Ryan, 2004), rapidly equilibrating with the alkaline $(\mathrm{pH} \sim 7.4)$ extracellular medium. 
This relief from proton quench leads to a fluorescence increase of $\sim 20$-fold. SpH thereafter is removed from the plasma membrane by internalization of synaptic vesicles whose fluorescence relaxes to baseline levels as they reacidify.

Previous work in our laboratory suggested that the slow decay $(\tau \sim 60 \mathrm{~s})$ of $\mathrm{SpH}$ fluorescence after a long stimulus train reflects the kinetics of endocytosis (Sankaranarayanan and Ryan, 2000). This work rested on the assumption that synaptic vesicle reacidification kinetics is sufficiently rapid with respect to internalization kinetics that reacidification may be safely ignored in interpreting $\mathrm{SpH}$ fluorescence transients. Although this was a reasonable assumption with the long stimulus trains that were used in previous work, here we are interested in short stimulus trains that preferentially mobilize the RRP. Previous work in our laboratory showed that internalization kinetics accelerates as we shorten the stimulus, leading to our concern that, after brief trains, it would be no longer safe simply to assume that reacidification kinetics could be safely discounted.

In principle, instantaneous presynaptic terminal $\mathrm{SpH}$ fluorescence can be separated into three components. The first derives from internal $\mathrm{SpH}$ quenched in acidic synaptic vesicles: the "acidic pool." The second derives from unquenched $\mathrm{SpH}$ exposed to the alkaline extracellular medium: the "surface pool." The third derives from SpH partially quenched in recently internalized synaptic vesicles that have not yet fully reacidified: the "alkaline pool." Exocytosis (whether classical or via a transient fusion pore) causes a rapid rise in $\mathrm{SpH}$ fluorescence, as the vesicles move from the acidic to the surface pool. Internalization (whether by classical endocytosis or by other mechanisms) is the movement of vesicles from the surface to the alkaline pool. $\mathrm{SpH}$ fluorescence is not diminished by internalization per se; fluorescence decays by proton quench as internalized vesicles in the alkaline pool reacidify, returning to the acidic pool.

The decay of $\mathrm{SpH}$ fluorescence after a short train is thus controlled by two factors: reacidification kinetics and internalization kinetics. We reasoned that, if we measured both reacidification kinetics and the time course of expansion and contraction of the alkaline pool, we could deconvolve the reacidification time course to estimate the internalization time course.

Our goal, then, was to devise ways to measure both reacidification kinetics and alkaline pool dynamics. If successful, we would be in a position to estimate internalization kinetics after short trains that selectively mobilize the RRP. This would directly test the hypothesis that rapid internalization is characteristic of RRP mobilization in small CNS terminals.

\section{Measurement of reacidification kinetics}

To measure reacidification kinetics, our strategy was to monitor fluorescence in single presynaptic boutons stimulated with brief trains, and then to quench surface $\mathrm{SpH}$ for $15 \mathrm{~s}$ by local pressurepulse application of acid. SpH in the alkaline pool (i.e., in recently endocytosed, partially reacidified vesicles) will be protected from this 15 s surface quench. $\mathrm{SpH}$ in fully acidified ( $\mathrm{pH}$ 5.5) vesicles will contribute a component of fluorescence detected during this period, but this component should be nearly constant. The decaying component of fluorescence during the quench should be a measure of reacidification of the alkaline pool.

As shown in Figure 1, we were able to detect fluorescence in line-scan mode from single boutons at a high temporal resolution (50 $\mathrm{Hz}$ sampling). A 5-s-long prestimulus acid quench $\left(Q_{0}\right)$ showed that the majority (typically $85-95 \%$ ) of resting fluorescence was attributable to surface $\mathrm{SpH}$, but a small, constant quench-resistant fraction remained, corresponding to quench-
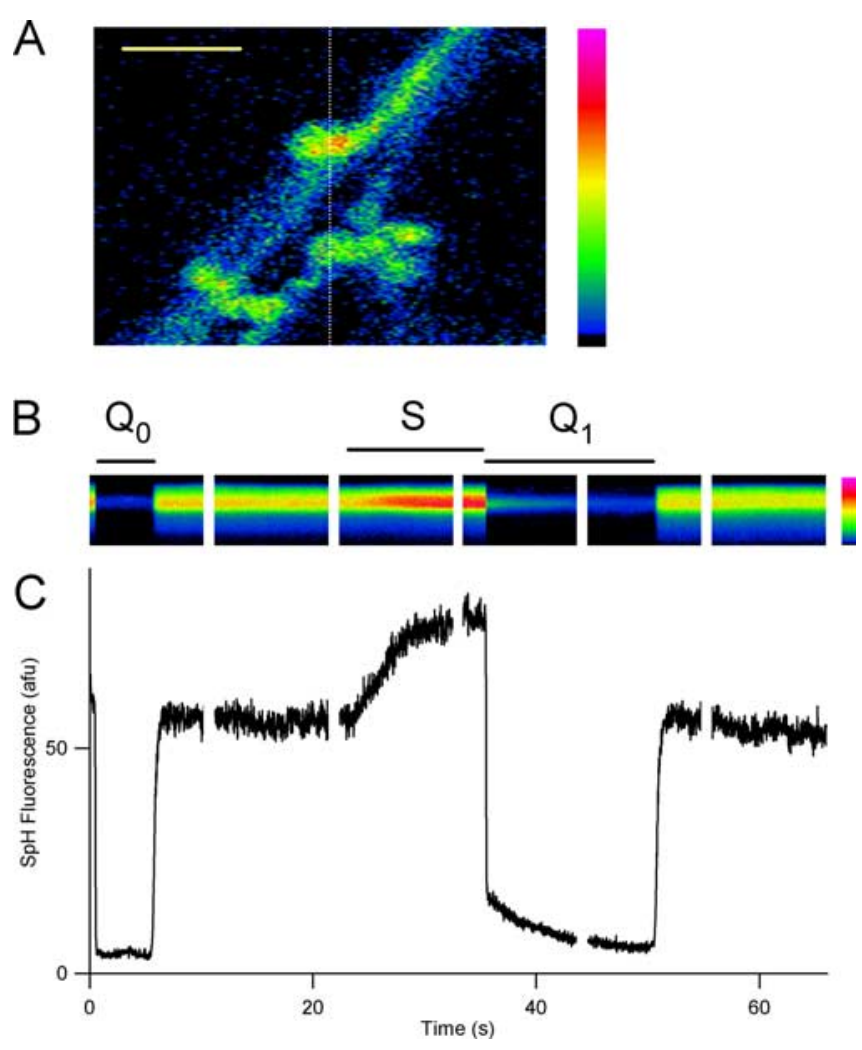

Figure 1. Rapid acid quench of surface $\mathrm{SpH}$ fluorescence allows measurement of internal alkaline vesicle pool and postendocytic reacidification time course. $\boldsymbol{A}$, Confocal image of resting fluorescence of axons and presynaptic terminals transfected with synapto-pHluorin, with a dashed line at the position of the scan line for $\boldsymbol{B}$. Scale bar, $3.4 \mu \mathrm{m}$. $\boldsymbol{B}$, Line-scan time series of fluorescence from a single bouton, with rapid surface quenching by pulsed-pressure application of impermeant acid ( 45 mm MES, pH 5.25) before $\left(Q_{0}, 5 \mathrm{~s}\right.$ duration) and immediately after $\left(Q_{1}\right.$, $15 \mathrm{~s})$ field electrical stimulation $(S ; 12 \mathrm{~s}, 20 \mathrm{~Hz})$. $\boldsymbol{A}, \boldsymbol{B}$, Pseudocolor scale bars (right) are linear, with black indicating zero fluorescence. $\boldsymbol{C}$, Plot of fluorescence time course, computed by averaging those rows from $\boldsymbol{B}$ with stimulus-evoked fluorescence increase. Time scale is the same as that in $\boldsymbol{B}$.

protected pools of $\mathrm{SpH}$. The quenching of surface fluorescence was very rapid, with a first-order time constant $\tau_{\mathrm{q}}$ of $32 \mathrm{~ms}$; in all such experiments accepted for additional analysis, $\tau_{\mathrm{q}}$ ranged in the tens of milliseconds (see Materials and Methods). After termination of the prequench, surface $\mathrm{SpH}$ rapidly re-equilibrates with the alkaline medium, and fluorescence recovers. During the stimulation period $(S)$, fluorescence increased as vesicular $\mathrm{SpH}$ was added to the surface. The poststimulus quench $\left(Q_{1}\right)$ revealed a much larger quench-resistant fluorescence signal than that during $Q_{0}$. This transient had a time course well approximated by a monoexponential decay with a time constant of $5.0 \mathrm{~s}$ and a baseline of 5.1 arbitrary fluorescence units (afu), slightly higher than the prequench mean fluorescence of $4.5 \mathrm{afu}$.

If the gradually declining fluorescence signal observed during the poststimulus quench $\left(Q_{1}\right)$ represents the reacidification kinetics of the alkaline pool, its decay should be blocked by Baf (Sankaranarayanan and Ryan, 2001). In Figure $2 A$, we show a representative experiment in which brief exposure to $1 \mu \mathrm{M} \mathrm{Baf}$ completely blocked the decay of fluorescence seen previously during the quench period. In similar experiments on eight different preparations stimulated for $30 \mathrm{~s}$ at $10 \mathrm{~Hz}$, the time courses of quench period fluorescence in control conditions were well fit by monoexponential decays with a mean time constant (mean \pm SEM) of $4.4 \pm 0.4 \mathrm{~s}(n=12)$ and a normalized amplitude of $1 \pm$ $0.13 \mathrm{U}(n=12)$. After Baf treatment, fluorescence during the 


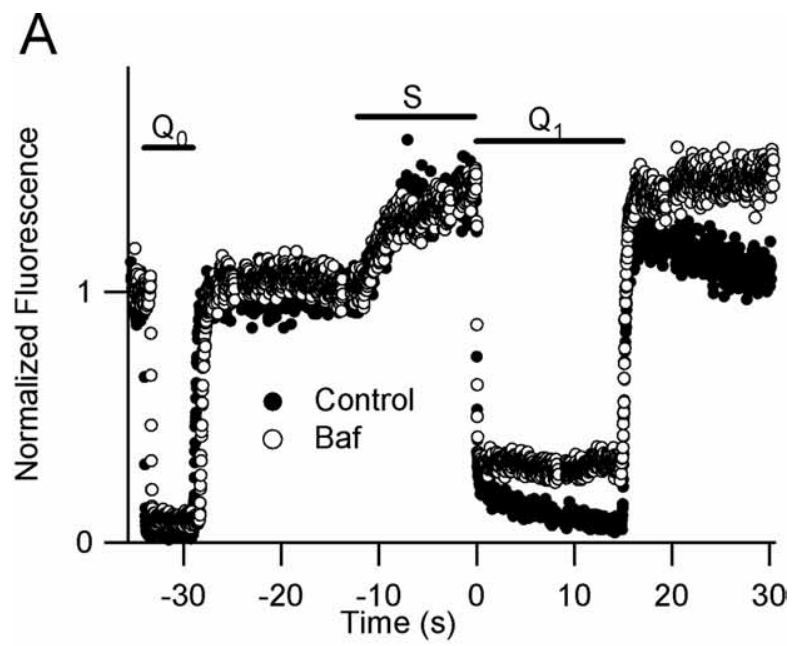

\section{B1}

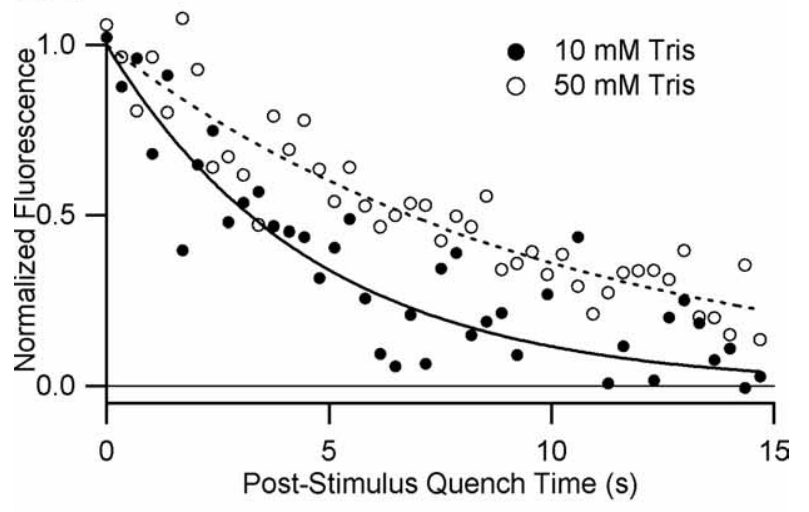

\section{B2}

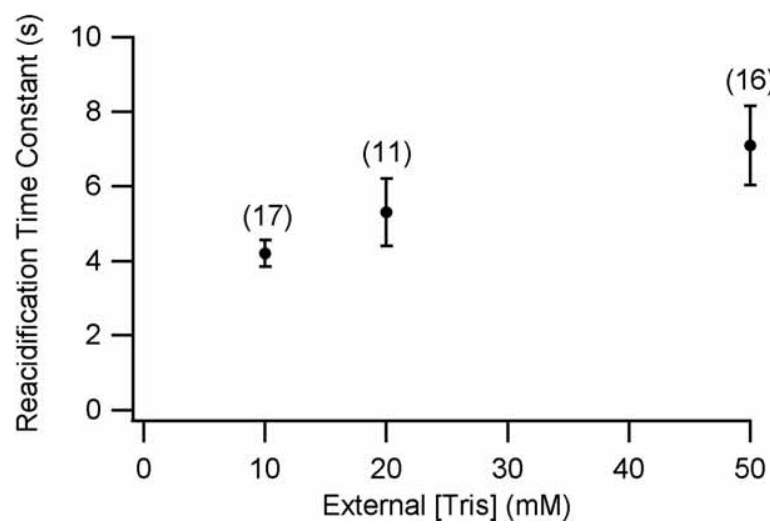

Figure 2. $\quad A, S p H$ fluorescence decay during poststimulus acid quench is blocked by a specific inhibitor of V-ATPase. SpH fluorescence sampled every $21 \mathrm{~ms}$ from a presynaptic bouton in control solution (closed circles) and after 30 s treatment with $1 \mu \mathrm{m}$ Baf (open circles). Pressurepulse application of impermeant acid before $\left(Q_{0}, 5 \mathrm{~s}\right)$ and immediately after $\left(Q_{1}, 15 \mathrm{~s}\right)$ a stimulus train $(S, 12 \mathrm{~s}, 20 \mathrm{~Hz})$ reveals a stimulation-dependent quench-resistant fluorescence transient whose decay is blocked by inhibition of the V-ATPase. Similar results were observed in a series of 13 paired trials on 8 separate preparations using a stimulus of $30 \mathrm{~s}$ at $10 \mathrm{~Hz}$ and a sampling interval of $342 \mathrm{~ms}$. $\boldsymbol{B}$, Sensitivity of reacidification kinetics to extracellular proton buffer. $\boldsymbol{B} \mathbf{1}$, Two fluorescence transients from the poststimulus quench periods of successive trials at the same bouton, normalized to unitary amplitude $\mathbf{B} \mathbf{2}$, Summary plot of reacidification time constants (mean \pm SEM) versus external [Tris]. Parentheses indicate number of trials for each point. All Tris experiments were performed in $91 \mathrm{~mm} \mathrm{NaCl}$ (see Materials and Methods) with stimulus trains of $30 \mathrm{~s}$ at $10 \mathrm{~Hz}$. Unpaired two-tailed Student's $t$ tests indicate a statistically significant difference ( $p<0.013$ ) between mean time constants in 10 and $50 \mathrm{~mm}$ Tris but no significant difference between 10 and $20 \mathrm{~mm}$ Tris $(p>0.19)$ or between 20 and $50 \mathrm{~mm}$ Tris $(p>0.23)$. quench period did not decay. Instead, quench period fluorescence gradually increased in intensity, with a mean slope of 1.4E$2 \pm 1.1 \mathrm{E}-2 \mathrm{U} / \mathrm{s}(n=8)$. In addition, the baseline fluorescence both before and during the quench is slightly elevated in the presence of bafilomycin. Both of these features are most likely attributable to slow alkalinization of synaptic vesicles, arising from either spontaneous exocytosis or a small proton leak from the vesicles. The effectiveness of Baf in blocking the fluorescence transient strongly supports the idea that the quench period fluorescence transient is a valid measure of reacidification kinetics.

To test whether the external $\mathrm{pH}$ buffer distorts reacidification kinetics, we varied external HEPES concentration. For 5 s $20 \mathrm{~Hz}$ stimuli, the poststimulus quench-resistant fluorescence decay time course was indifferent to external HEPES concentration between 5 and $40 \mathrm{~mm}$ (data not shown). For these experiments, time constants of decay were $3.8 \pm 0.4 \mathrm{~s}(5 \mathrm{mM}$ HEPES; $n=9), 4.3 \pm$ $0.3 \mathrm{~s}(25 \mathrm{~mm} ; n=5)$, and $4.0 \pm 0.4 \mathrm{~s}(40 \mathrm{~mm} ; n=9)$. In several of these experiments, solution changes were followed by a long train of electrical stimulation $(90 \mathrm{~s}, 10 \mathrm{~Hz})$, to test whether extensive endo-exo cycling favors access of HEPES to the vesicle lumina, as reported previously (Gandhi and Stevens, 2003). However, we found no effect of vesicle turnover on reacidification kinetics; it appears likely that either HEPES is excluded from vesicles undergoing "compensatory" endocytosis or that it is ineffective at proton buffering within the microenvironment of the synaptic vesicle lumen.

We also tested the effect of external Tris on reacidification kinetics (Fig. 2B1,B2). To facilitate comparison with previous work (Gandhi and Stevens, 2003), we used a low $\mathrm{Na}^{+}$Tyrode's solution for these experiments (see Materials and Methods). After a $30 \mathrm{~s}, 10 \mathrm{~Hz}$ stimuli we found that high Tris slows the decay of the reacidification transient (Fig. $2 \mathrm{B1}$ ). In a series of such experiments on nine individual boutons from six separate preparations, reacidification time constants (mean \pm SEM) of $4.2 \pm 0.35$ $(n=17), 5.3 \pm 0.90(n=11)$, and $7.1 \pm 1.1 \mathrm{~s}(n=16)$ were observed with 10, 20, and $50 \mathrm{~mm}$ Tris, respectively (Fig. 2B2). Unpaired two-tailed Student's $t$ tests indicate a statistically significant difference $(p<0.013)$ between mean time constants in 10 and $50 \mathrm{~mm}$ Tris. Thus, as reported previously, external Tris slows reacidification, most likely by entering nascent vesicles and augmenting their buffering capacity.

To test whether photobleaching of the alkaline pool distorts the fluorescence time course, we performed surface-quenching experiments while varying the line-scan sampling rate from 1 to $50 \mathrm{~Hz}$. We saw no change in the time course of fluorescence decay (data not shown).

The observed sensitivity of $\mathrm{SpH}$ fluorescence transients during the quench period to Baf and to external Tris strongly supports the idea that these signals represent postinternalization reacidification kinetics.

Subsequently, we wished to test whether there was a difference between reacidification kinetics of vesicles in the RRP versus the recycling pool (RP). In our system, a 40 ap, $1 \mathrm{~s}$ stimulus mobilizes only $9.1 \pm 0.7 \%$ of the total recycling pool (102 boutons in four separate experiments; data not shown), whereas a $300 \mathrm{ap}, 10 \mathrm{~s}$ stimulus mobilizes $\sim 75 \%$ (Ryan et al., 1996). Thus, we expect short trains selectively to mobilize the RRP, whereas longer trains will mobilize both RRP and the entire RP. We found that for both short and long trains, reacidification time course during the $15 \mathrm{~s}$ quench period was well approximated by a monoexponential decay. Similar time constants were observed for experiments with $40 \mathrm{APs}$ at $40 \mathrm{~Hz}(4.5 \pm 1.2 ; n=4), 100$ at $20 \mathrm{~Hz}(4.3 \pm 0.3 ; n=5)$, 120 at $20 \mathrm{~Hz}(4.8 \pm 0.1 ; n=2), 240$ at $20 \mathrm{~Hz}(4.8 \pm 0.5 ; n=14)$, 
and 300 at $10 \mathrm{~Hz}(4.4 \pm 0.4 ; n=12)$. These experiments suggest that vesicles in the RRP and the RP share similar reacidification kinetics.

\section{Measurement of alkaline pool dynamics}

Having measured the time course of reacidification after RRP mobilization, we next sought to measure alkaline pool dynamics, with a view toward eventually estimating internalization kinetics. We noted that alkaline pool amplitude at time $\Delta t$ after stimulus onset is proportional to the initial amplitude of the quenchresistant $\mathrm{SpH}$ fluorescence transient. We performed experiments in which the interval between stimulus and quench onsets, $\Delta t$, was varied between 1 and $20 \mathrm{~s}$, and the size of the alkaline pool was measured at each $\Delta t$ (for details, see Materials and Methods).

In two successive trials from a representative experiment (Fig. $3 A)$, quenching immediately after a $1 \mathrm{~s}, 40 \mathrm{~Hz}$ train $(\Delta t=1 \mathrm{~s})$ revealed a small alkaline pool that decayed exponentially. Delaying the quench $(\Delta t=8 \mathrm{~s})$ revealed a larger alkaline pool that also decayed exponentially. In a representative experiment (Fig. $3 B$, top) with nine such trials in the same bouton, the plot of alkaline pool size versus $\Delta t$ resembled an $\alpha$ function (see Materials and Methods) with time constant (mean \pm SD) of $4.6 \pm 1 \mathrm{~s}$ and time intercept of $0.31 \pm 0.49 \mathrm{~s}$. Data from 10 such experiments (Fig. $3 B$, bottom), with 5-10 trials in each bouton, shows that, after a $1 \mathrm{~s}, 40 \mathrm{~Hz}$ train, alkaline pool dynamics may be approximated by an $\alpha$ function with a time constant of $6.4 \pm 0.95 \mathrm{~s}$ (mean $\pm \mathrm{SD}$ ) and a time intercept at $0.05 \pm 0.42 \mathrm{~s}$. We note that the appearance of a small but measurable alkaline pool in trials with $\Delta t=1 \mathrm{~s}$ argues against any significant delay in the onset of internalization after these stimuli.

In summary, after brief stimulus trains, the fluorescence of the alkaline pool peaks $\sim 6 \mathrm{~s}$ after the stimulus and then slowly decays over tens of seconds. With these measurements of alkaline pool dynamics and with our measurements of reacidification kinetics, we were now in a position to use deconvolution to estimate the time course of internalization of synaptic vesicles after brief trains.

\section{Alkaline pool deconvolution and simulation}

If we assume that the processes governing alkaline pool dynamics are linear and time invariant (see Materials and Methods), we can deconvolve the output process (reacidification time course) from alkaline pool dynamics to estimate the input process (endocytosis time course). Deconvolution (Fig. 4) allowed us to estimate the time course of endocytosis after a $1 \mathrm{~s}, 40 \mathrm{~Hz}$ train as a curve whose shape is closely approximated by an exponential decay with a time constant of $7.6 \mathrm{~s}$. Notably, there is no indication of a very rapid component of endocytosis, as would be expected if a significant fraction of vesicles were undergoing rapid reuse.

To estimate the relative contributions of surface and alkaline pools to the fluorescence transients observed after a brief train, we made a very simple numerical simulation (Fig. 5) that implemented the following scheme:

$$
k_{\text {exo }} \quad k_{\text {endo }} \quad k_{\text {reacid }}
$$

Releasable pool $\rightarrow$ surface pool $\rightarrow$ alkaline pool $\rightarrow$ acidic pool.

The rate constants $k_{\text {exo }}$ and $k_{\text {endo }}$ describe the kinetics of exocytosis and endocytosis; $k_{\text {reacid }}$ is the rate constant of vesicle reacidification. We modeled exocytosis as a zero-order reaction with rate constant $k_{\text {exo }}=1 \mathrm{~s}^{-1}$. Our estimate of $k_{\text {reacid }}$ was $0.2 \mathrm{~s}^{-1}$, the reciprocal of the time constant of reacidification. We then searched for a value of $k_{\text {endo }}$ such that the simulated time course
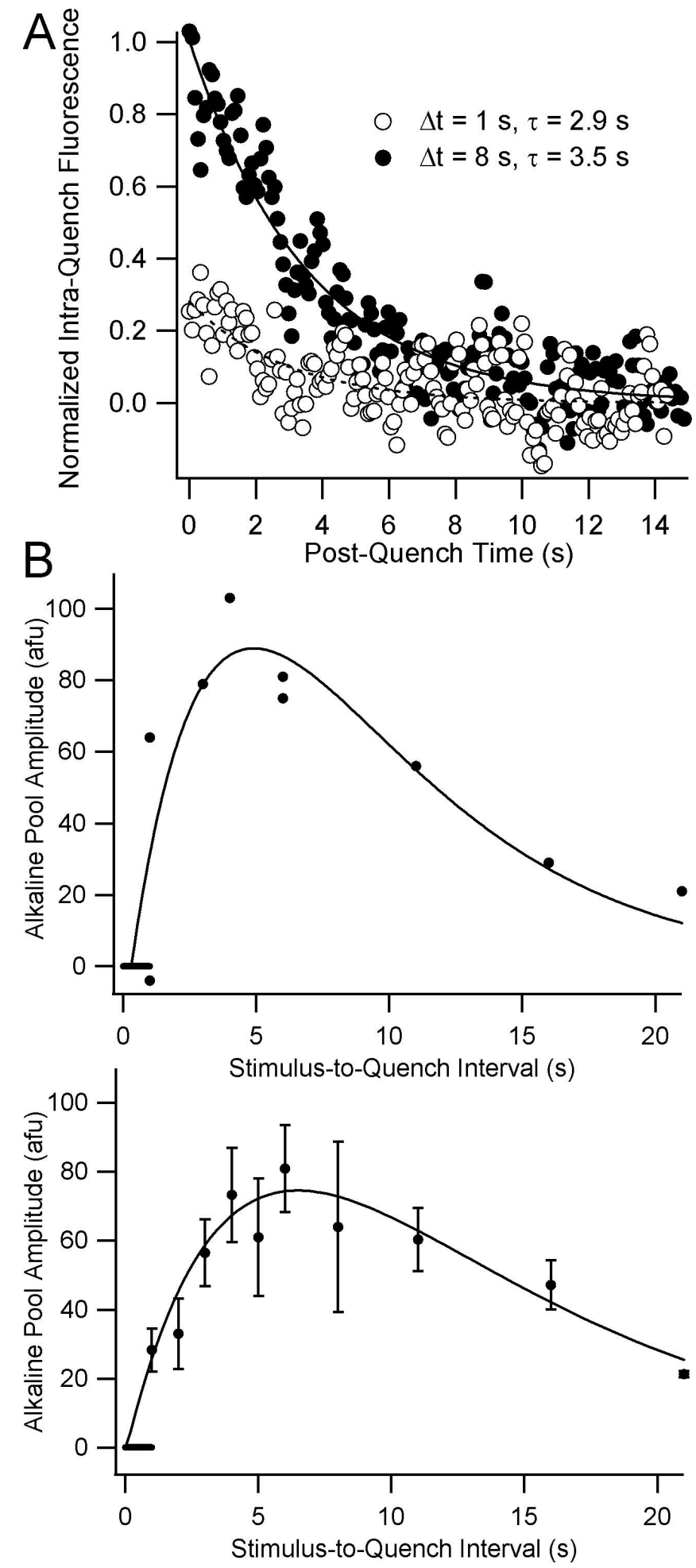

Figure 3. Time course of alkaline pool growth and decay after a $1 \mathrm{~s}, 40 \mathrm{~Hz}$ train. $A$, Normalized fluorescence transients measured during $15 \mathrm{~s}$ quenches begun $1 \mathrm{~s}$ and $8 \mathrm{~s}$ after stimulus onset. The alkaline pool amplitude at time $\Delta t$ is estimated by taking the difference of the means of the first five and the last five points of the quench period. Sampling interval for these experiments is $85.4 \mathrm{~ms}$. B, Plots of alkaline pool amplitudes from a representative experiment (top) and from seven such experiments (bottom; mean \pm SEM) versus the interval between stimulus and quench onsets. Curves are fits to an $\alpha$ function of the following form: $y=k^{*}(\Delta t-$ delay) $)^{*} \exp (-(\Delta t-$ delay $) / \tau)$, where $k$ is a scaling factor, and $\Delta t$ is the stimulus - quench interval, and delay is a time offset term. The fit parameters $\{k$, delay, $\tau\}( \pm$ SD) were $\{53 \pm 16$, $0.3 \pm 0.5 \mathrm{~s}, 4.6 \pm 1.0 \mathrm{~s}\}$ for the curve in the top panel and $\{31 \pm 6,0.1 \pm 0.4 \mathrm{~s}, 6.4 \pm 1.0 \mathrm{~s}\}$ for the curve in the bottom panel (see Materials and Methods). Horizontal bars at origins indicate the stimulus period. 


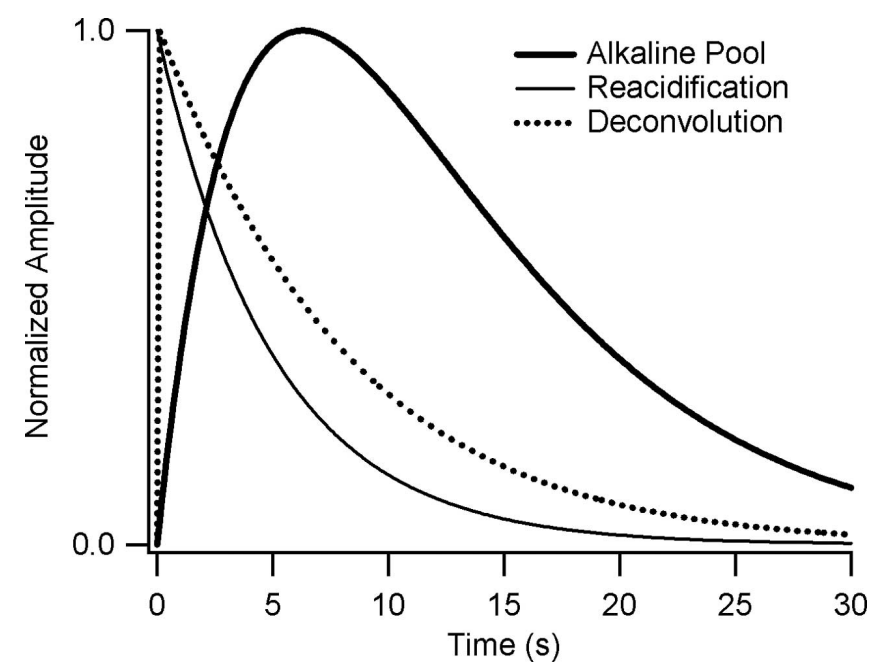

Figure 4. Deconvolution of reacidification time course (thin solid line) from alkaline pool dynamics (thick solid line) gives the estimated time course of endocytosis (dotted line) after a $1 \mathrm{~s}, 40 \mathrm{~Hz}$ train. Each curve was normalized to its peak value.

of the alkaline pool matched the average alkaline pool time course we measured after a $1 \mathrm{~s}, 40 \mathrm{~Hz}$ stimulus. We found that a rate constant of $0.135 \mathrm{~s}^{-1}$ gave a good match to the time course of the alkaline pool (Fig. 5A). These rate constants for endocytosis and reacidification imply a relative amplitude of $\sim 0.3$ for the peak of the alkaline pool, where 1 corresponds to the amount of $\mathrm{SpH}$ exocytosed during a $1 \mathrm{~s}, 40 \mathrm{~Hz}$ train.

This simulation helps to illustrate the importance of considering alkaline pool dynamics in interpreting rapidly decaying $\mathrm{SpH}$ transients. In Figure $5 B$, the predicted total $\mathrm{SpH}$ fluorescence decays with a time constant of $\sim 11 \mathrm{~s}$ and closely matches a measured SpH transient after a $1 \mathrm{~s}, 40 \mathrm{~Hz}$ stimulus (Fig. 5C). $\mathrm{SpH}$ internalization occurs with a time constant of $\sim 7.4 \mathrm{~s}$. Ignoring alkaline pool dynamics would have led to a $50 \%$ overestimate of the time constant of internalization.

\section{Discussion}

To understand vesicle refilling and quantal size determinants during ongoing activity, we set out to study synaptic vesicle reacidification kinetics by monitoring the fluorescence of $\mathrm{SpH}$ in CNS boutons after trains of APs. By rapidly quenching surface $\mathrm{SpH}$ with pressure-pulse applications of acid, we isolated a quench-protected fluorescence signal that reflects reacidification kinetics isolated from distortion by ongoing endocytosis. Surface quenching also eliminates alternate fluorescence decay pathways, such as that which might be caused by lateral diffusion of vesicular $\mathrm{SpH}$ from sites of exocytosis into neighboring axon (Sankaranarayanan and Ryan, 2000). A possible artifact of quench experiments could arise from slow access of the acid to surface $\mathrm{SpH}$ in tortuous membrane invaginations, posited by some (Wilkinson and Cole, 2001) to arise in the poststimulus period. The complete block of the decay of fluorescence during the quench period by exposure to the V-ATPase inhibitor Baf (Fig. 2) demonstrates that these fluorescence transients reflect reacidification kinetics rather than lateral diffusion of $\mathrm{SpH}$ or slow quench access. To our knowledge, these are the first direct measurements in intact terminals of postendocytic reacidification kinetics, unambiguously isolated from the kinetics of endocytosis and that of lateral diffusion of $\mathrm{SpH}$ in the plasma membrane.

Our principle findings are as follows: (1) rapid acid quench of surface $\mathrm{SpH}$ may be used to isolate fluorescence from the alkaline
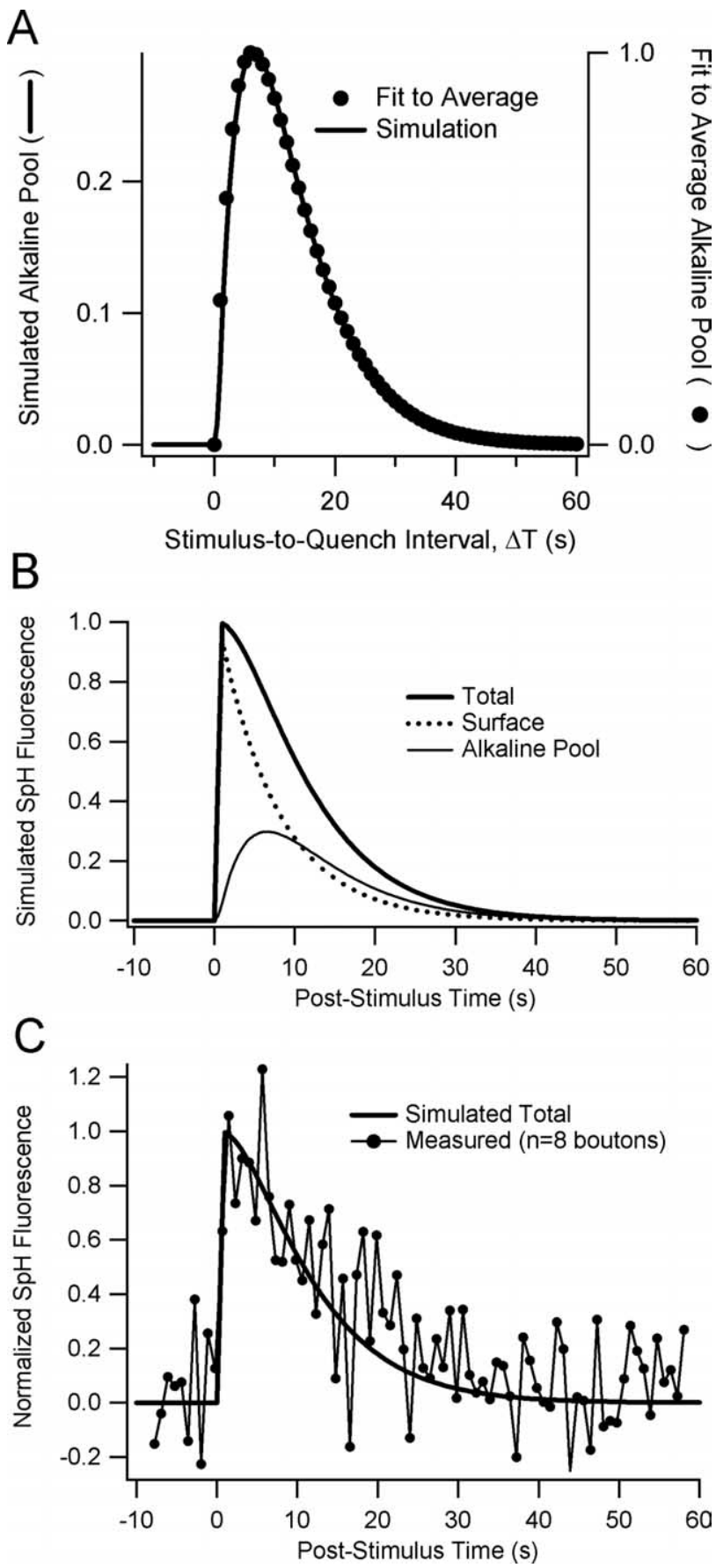

Figure 5. Simple simulation of alkaline pool dynamics. A, Superposition of average alkaline pool time course (closed circles) and simulated alkaline pool time course (solid line). Numerical simulation used a zero-order rate constant of $1 \mathrm{~s}^{-1}$ for exocytosis and first-order rate constants of 0.135 and $0.2 s^{-1}$ for endocytosis and reacidification, respectively. $\boldsymbol{B}$, Time courses of surface (dotted line) and alkaline pool (thin solid line) SpH fluorescence; their sum is the expected SpH fluorescence transient (thick solid line) with no surface quenching. The time course of reacidification significantly influences the time course of $\mathrm{SpH}$ fluorescence transient after brief trains. $\boldsymbol{C}$, The simulated $\mathrm{SpH}$ curve (smooth line) from $(\boldsymbol{B})$ with superimposed data from an experiment with $1 \mathrm{~s}, 40 \mathrm{~Hz}$ stimulation and field scanning of eight boutons with sampling interval $\sim 700$ ms. Error bars were omitted for clarity.

pool of reacidifying vesicles and to measure directly the kinetics of reacidification; (2) after compensatory endocytosis, reacidification of synaptic vesicles occurs with apparent first-order kinetics and a time constant of 4-5 s; (3) similar reacidification kinetics are observed after a variety of stimulus pulse protocols; (4) 
external $\mathrm{pH}$ buffers (Tris but not HEPES) can slow such reacidification kinetics; (5) by varying the stimulus-to-quench interval, the growth and decay of the alkaline pool may be estimated after brief stimuli; and (6) the time course of endocytosis after brief trains, otherwise very difficult to measure in small presynaptic terminals, may be estimated by deconvolution of reacidification kinetics from alkaline pool dynamics.

Our most important finding is that postendocytic reacidification, after trains of 40-300 AP, occurs quite slowly, with a time constant of 4-5 s. Reacidification on this time scale is not expected to contribute significantly to the time course of $\mathrm{SpH}$ fluorescence decay (time constants, 20-80 s) after trains longer than $\sim 60 \mathrm{AP}$ at $10 \mathrm{~Hz}$ (Sankaranarayanan and Ryan, 2000). For such trains, $\mathrm{SpH}$ fluorescence decay may be safely interpreted as reflecting the time course of endocytosis. However, after brief trains, $\mathrm{SpH}$ fluorescence transients decay more rapidly, on a time scale similar to reacidification; their decay times result from a combination of endocytosis and reacidification kinetics. We found that, by varying the stimulus-to-quench interval, we could measure alkaline pool dynamics (Fig. 3), from which we deconvolved reacidification kinetics to estimate endocytosis kinetics (Fig. 4). After a $1 \mathrm{~s}, 40 \mathrm{~Hz}$ stimulus, surface $\mathrm{SpH}$ is internalized with a time constant of $\sim 7.6 \mathrm{~s}$. It is important to note that it would be invalid to try to estimate endocytosis rates by deconvolving reacidification from the unquenched $\mathrm{SpH}$ fluorescence transient, which includes fluorescence both from internalized, reacidifying compartments and from surface $\mathrm{SpH}$ not subject to reacidification.

Our approach has allowed us to test the hypothesis that, in small CNS presynaptic terminals, mobilization of the vesicles of the RRP by short trains of electrical stimulation leads to "kissand-run" cycling with rapid internalization and presumably rapid reuse of vesicles, enabling the synapse to avoid exhaustion of the RRP and to maintain signaling capacity. Evidence for kissand-run cycling at central synapses has emerged from a variety of experiments based on destaining of amphipathic probes that differ significantly in their conclusions about both the fusion-tofission time scale as well as the conditions under which kiss-andrun will dominate (Ryan et al., 1996; Klingauf et al., 1998; Pyle et al., 2000; Stevens and Williams, 2000; Zenisek et al., 2002; Aravanis et al., 2003; Richards et al., 2005) However, definitive conclusions based on FM destaining characteristics are difficult to achieve because the detailed physical behavior of the dye within the confines of the vesicle, such as whether it dissociates from the vesicular membrane with the same dissociation constant as that from the plasma membrane, are not known.

Alternative approaches to examining the details of fusion-tofission part of the vesicle cycle have become possible with the use of $\mathrm{SpH}$, a modified GFP tethered to a synaptic vesicle protein. These experiments exploit the fact that $\mathrm{SpH}$ has a proton-binding site whose occupancy reversibly quenches $\mathrm{SpH}$ fluorescence by $\sim 20$-fold; this site is in rapid equilibrium with protons in the vesicle lumen or at the cell surface, allowing $\mathrm{SpH}$ to rapidly signal synaptic vesicle fusion with the plasma membrane or changes in synaptic vesicle $\mathrm{pH}$. Gandhi and Stevens (2003) measured small fluorescence transients in SpH-transfected hippocampal neurons after a single AP. These transients were interpreted as representing the fusion, uptake, and reacidification of individual synaptic vesicles. The authors propose a scheme in which there are three modes of internalization after single APs: fast (or kiss-and-run), slow (or compensatory), and triggered (or "stranded"). Reacidification of vesicles internalized by the fast or triggered modes was found to be very rapid, with a time constant of $\sim 500 \mathrm{~ms}$. In this study, the ratio of kiss-and-run events to compensatory endocytosis was highly dependent on the release probability, was skewed toward kiss-and-run only for very low release probability conditions, and was only tested for a small number of stimuli at very long interpulse intervals. Furthermore, the interpretation of the data in that work, in particular the fluorescence decreases after exocytosis, implicitly assumed that synaptic vesicle proteins do not escape the active zone by lateral diffusion. Postexocytosis lateral diffusion of $\mathrm{SpH}$, which would also lead to fluorescence decreases have been shown to occur in these synapses (Sankaranarayanan and Ryan, 2000) and would be even more prevalent under conditions in which the surface molecules were photobleached before stimulation. All of these difficulties underscore the need to use means that discriminate against surface versus internal fluorescence when interpreting $\mathrm{SpH}$ signals.

Using such means, we found that, after a $1 \mathrm{~s}, 40 \mathrm{~Hz}$ stimulus that preferentially mobilizes the RRP, surface $\mathrm{SpH}$ is internalized with a time constant of $\sim 7.6 \mathrm{~s}$. There was no sign of rapid internalization, arguing against rapid reuse. This time scale is consistent with the saturation model that we originally proposed for these nerve terminals (Sankaranarayanan and Ryan, 2000), whereby the time constant for vesicle retrieval after the stimulus is proportional to the amount of accumulated membrane during stimulation and agrees well with the recent findings that vesicles that comprise the RRP are not preferentially reused after exocytosis ( $\mathrm{Li}$ et al., 2005). The continuous progression of endocytosis over a several second time period, even after brief stimuli expected to mobilize the RRP, but not the RP, is not consistent with the recent suggestion that, although RP vesicles may use the "slow" classical cycling pathway, RRP vesicle cycling occurs predominantly by the "fast" kiss-and-run pathway. It might be argued that kiss-and-run cycling with rapid internalization is occurring in our system but that we are insensitive to these events because the "kiss" is so ephemeral that protons do not have a chance to equilibrate across the fusion pore. This seems unlikely for three reasons. First, if the fusion pore were so short-lived that protons did not equilibrate, it would be unable to release a significant quantity of neurotransmitter. Second, Gandhi and Stevens (2003) report quantal SpH signals, which suggest but do not imply full pH equilibration. Third, Aravanis et al. (2003) argue for a mean fusion pore lifetime on the order of $1 \mathrm{~s}$ after short trains.

Thus, our data impose significant restrictions on the degree to which a rapid kiss-and-run type mechanism of internalization occurs at these nerve terminals and argue strongly against the predominance of this pathway after rapid mobilization of the RRP. These results agree well with recent measurements of membrane retrieval at the calyx of Held, which also argue against a kiss-and-run mechanism during repetitive stimulation at that synapse (Yamashita et al., 2005). Our measurements do not lend themselves well to measurements at very-low-frequency stimulation, and thus it is possible that, only under those conditions, kiss-and-run would dominate, which would be consistent with recent measurements of subquantal dye destaining using lowfrequency stimulation (Aravanis et al., 2003; Richards et al., 2005).

It is important to note that, although accurate measurement of the kinetics of synaptic vesicle reacidification will be crucial for understanding how vesicles are readied for reuse, the precise relationship between vesicle reacidification and refilling kinetics remains to be determined (Tabb et al., 1992; Wolosker et al., 1996; Huang et al., 2001; Stobrawa et al., 2001; Sonawane et al., 2002; Vanoye and George, 2002; Robinson et al., 2004). 


\section{References}

Aravanis AM, Pyle JL, Tsien RW (2003) Single synaptic vesicles fusing transiently and successively without loss of identity. Nature 423:643-647.

Fernandez-Alfonso T, Ryan TA (2004) The kinetics of synaptic vesicle pool depletion at CNS synaptic terminals. Neuron 41:943-953.

Fremeau Jr RT, Voglmaier S, Seal RP, Edwards RH (2004) VGLUTs define subsets of excitatory neurons and suggest novel roles for glutamate. Trends Neurosci 27:98-103.

Gandhi SP, Stevens CF (2003) Three modes of synaptic vesicular recycling revealed by single-vesicle imaging. Nature 423:607-613.

Grabe M, Oster G (2001) Regulation of organelle acidity. J Gen Physiol 117:329-344.

Huang P, Liu J, Di A, Robinson NC, Musch MW, Kaetzel MA, Nelson DJ (2001) Regulation of human CLC-3 channels by multifunctional $\mathrm{Ca}^{2+}$ / calmodulin-dependent protein kinase. J Biol Chem 276:20093-20100.

Klingauf J, Kavalali ET, Tsien RW (1998) Kinetics and regulation of fast endocytosis at hippocampal synapses. Nature 394:581-585.

Miesenbock G, De Angelis DA, Rothman JE (1998) Visualizing secretion and synaptic transmission with $\mathrm{pH}$-sensitive green fluorescent proteins. Nature 394:192-195.

Li Z, Li Z, Burrone J, Tyler WJ, Hartman KN, Albeanu DF, Murthy VN (2005) Synaptic vesicle recycling studied in transgenic mice expressing synaptopHluorin. Proc Natl Acad Sci USA 102:6131-6136.

Liu Y, Edwards RH (1997) The role of vesicular transport proteins in synaptic transmission and neural degeneration. Annu Rev Neurosci 20:125-156.

Nishi T, Forgac M (2002) The vacuolar (H+)-ATPases-nature's most versatile proton pumps. Nat Rev Mol Cell Biol 3:94-103.

Pothos EN, Mosharov E, Liu KP, Setlik W, Haburcak M, Baldini G, Gershon MD, Tamir H, Sulzer D (2002) Stimulation-dependent regulation of the $\mathrm{pH}$, volume and quantal size of bovine and rodent secretory vesicles. J Physiol 542:453-476.

Pyle JL, Kavalali ET, Piedras-Renteria ES, Tsien RW (2000) Rapid reuse of readily releasable pool vesicles at hippocampal synapses. Neuron 28:221-231.

Richards DA, Bai J, Chapman ER (2005) Two modes of exocytosis at hippocampal synapses revealed by rate of FM1-43 efflux from individual vesicles. J Cell Biol 168:929-939.

Robinson NC, Huang P, Kaetzel MA, Lamb FS, Nelson DJ (2004) Identification of an $\mathrm{N}$-terminal amino acid of the CLC-3 chloride channel critical in phosphorylation-dependent activation of a CaMKII-activated chloride current. J Physiol 556:353-368.

Ryan TA (1999) Inhibitors of myosin light chain kinase block synaptic vesicle pool mobilization during action potential firing. J Neurosci 19:1317-1323.

Ryan TA, Smith SJ, Reuter H (1996) The timing of synaptic vesicle endocytosis. Proc Natl Acad Sci USA 93:5567-5571.

Sankaranarayanan S, Ryan TA (2000) Real-time measurements of vesicleSNARE recycling in synapses of the central nervous system. Nat Cell Biol 2:197-204.

Sankaranarayanan S, Ryan TA (2001) Calcium accelerates endocytosis of vSNAREs at hippocampal synapses. Nat Neurosci 4:129-136.

Sonawane ND, Thiagarajah JR, Verkman AS (2002) Chloride concentration in endosomes measured using a ratioable fluorescent $\mathrm{Cl}^{-}$indicator: evidence for chloride accumulation during acidification. J Biol Chem 277:5506-5513.

Stevens CF, Williams JH (2000) "Kiss and run" exocytosis at hippocampal synapses. Proc Natl Acad Sci USA 97:12828-12833.

Stobrawa SM, Breiderhoff T, Takamori S, Engel D, Schweizer M, Zdebik AA, Bosl MR, Ruether K, Jahn H, Draguhn A, Jahn R, Jentsch TJ (2001) Disruption of ClC-3, a chloride channel expressed on synaptic vesicles, leads to a loss of the hippocampus. Neuron 29:185-196.

Tabb JS, Kish PE, Van Dyke R, Ueda T (1992) Glutamate transport into synaptic vesicles. Roles of membrane potential, $\mathrm{pH}$ gradient, and intravesicular pH. J Biol Chem 267:15412-15418.

Vanoye CG, George AL Jr (2002) Functional characterization of recombinant human ClC-4 chloride channels in cultured mammalian cells. J Physiol (Lond) 539:373-383.

Wilkinson RS, Cole JC (2001) Resolving the Heuser-Ceccarelli debate. Trends Neurosci 24:195-197.

Wolosker H, de Souza DO, de Meis L (1996) Regulation of glutamate transport into synaptic vesicles by chloride and proton gradient. J Biol Chem 271:11726-11731.

Yamashita T, Hige T, Takahashi T (2005) Vesicle endocytosis requires dynamin-dependent GTP hydrolysis at a fast CNS synapse. Science 307:124-127.

Zenisek D, Steyer JA, Feldman ME, Almers W (2002) A membrane marker leaves synaptic vesicles in milliseconds after exocytosis in retinal bipolar cells. Neuron 35:1085-1097. 